\title{
The Natural Alkaloid Berberine Can Reduce the Number of Pseudomonas aeruginosa Tolerant Cells
}

\author{
Gianmarco Mangiaterra $^{\dagger^{* * *}}$, Nicholas Cedraro ${ }^{\dagger * *}$, Emiliano Laudadio ${ }^{\ddagger}$, Cristina Minnelli \\ Barbara Citterio $^{\S}$, Francesca Andreoni§, Giovanna Mobbili ${ }^{\dagger}$, Roberta Galeazzi ${ }^{\dagger}$ and

\section{Francesca Biavasco ${ }^{\dagger}$}

†Department of Life and Environmental Sciences, Polytechnic University of Marche, via Brecce Bianche, 60131, Ancona, Italy.

Department of Sciences and Engineering of Matter, Environment and Urban Planning, Polytechnic University of Marche, via Brecce Bianche, 60131, Ancona, Italy

${ }^{\S}$ Department of Biomolecular Sciences, sect. Biotechnology, University of Urbino "Carlo Bo", Via Arco d'Augusto 2, 61032, Fano, Italy.

*Corresponding author, Department of Life and Environmental Sciences, Polytechnic University of Marche, via Brecce Bianche, 60131, Ancona, Italy, g.mangiaterra@pm.univpm.it, Phone: +39 071 220 4622, Fax: +390712204316

\section{Supplementary materials}

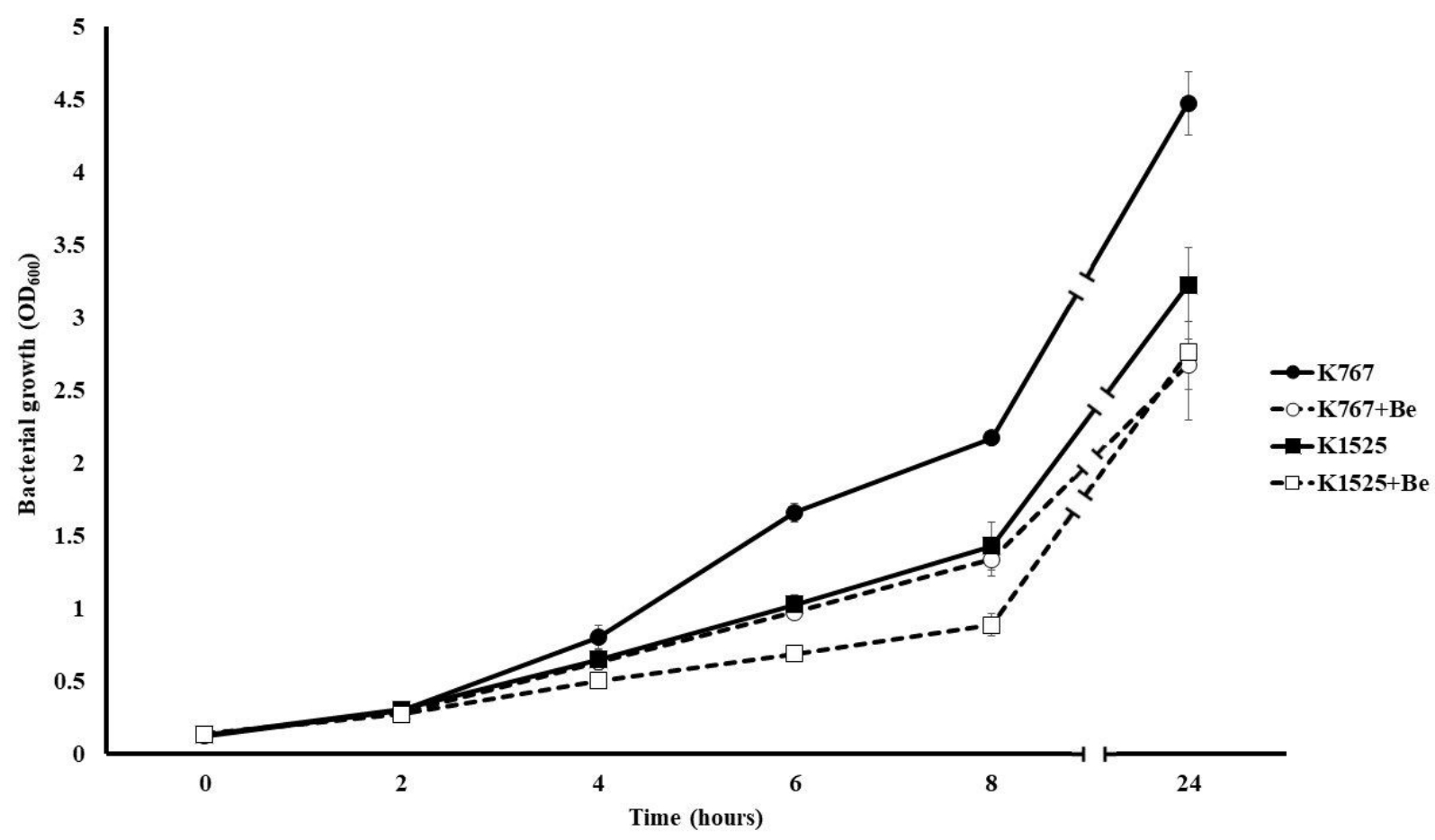

Figure S1. Influence of berberine on the growth rate of the isogenic pair P. aeruginosa K767 / K1525. Overnight cultures of the isogenic pair P. aeruginosa K767 / K1525 in LB broth were diluted to $\mathrm{OD}_{600}=0.1$ and incubated for 24 $\mathrm{h}$ at $37{ }^{\circ} \mathrm{C}$ with shaking $(140 \mathrm{rpm})$ in the absence / presence of berberine (Be) $80 \mu \mathrm{g} / \mathrm{ml}$. Bacterial abundance was detected after $2,4,6,8$ and $24 \mathrm{~h}$ by reading $\mathrm{OD}_{600}$. The results are the mean of 3 assays \pm standard deviation. 
>AB015853.1:1331-4471 Pseudomonas aeruginosa gene for MexX and MexY, complete cds

ATGGCTCGTT TCTTCATTGA CCGGCCGGTC TTCGCCTGGG TGATCTCCCT

GCTGATCGTG CTCGCCGGGG TCCTGGCGAT CCGCTTCCTG CCGGTCGCCC AGTACCCGGA CATCGCGCCG CCGGTGGTCA ACGTCAGCGC CACGTATCCC GGCGCCTCGG CCAAGGTGGT CGAGGAAGCG GTGACCGCGA TCATCGAGCG CGAGATGAAC GGCGCGCCCG GCCTGCTCTA CACCAAGGCC ACCAGCAGCA CCGGCCAGGC CTCGCTGACC CTGACCTTCC GCCAGGGCGT GAACGCGAAC CTCGCCGCGG TGGAAGTGCA GAACCGCCTG AAGATCGTCG AGTCGCGCCT GCCCGAATCG GTGCGGCGCG ACGGCATCTA CGTGGAGAAG GCGGCGGACA GCATCCAGCT GATCGTTACC CTTACCTCCT CCAGCGGCCG CTACGACGCC ATGGAGCTGG GCGAGATCGC CTCGTCCAAC GTGTTGCAGG CGCTGCG3CCG GGTGGAGGGC GTGGGCAAGG TCGAGACCTG GGGCGCCGAG TACGCCATGC GCATCTGGCC CGACCCGGCC AAGCTGACCT CGATGAACCT CAGCGCCAGC GACCTGGTCA ACGCCGTGCG CCGGCACAAC GCCCGCCTCA CCGTGGGCGA CATCGGCAAC CTCGGGGTCC CCGACTCGGC GCCGATCAGC GCCACGGTGA AGGTCGACGA CACCCTGGTG ACGCCCGAGC AGTTCGGCGA AATTCCGCTG GCGCATCCGC GCGACGGCGG CGCGATCCGC CTGCGCGACG TGGCCCGCGT CGAGTTCGGC CAGAGCGAGT ACGGCTTCGT CTCGCGGGTC AACCAAATGA CCGCCACCGG CCTGGCGGTG AAGATGGCGC CCGGCTCCAA CGCGGTGGCC ACCGCCAAGC GCATCCGCGC CACCCTCGAC GAGCTGTCGC GCTACTTCCC GGAGGGCGTG AGCTACAACA TCCCCTATGA CACCTCGGCG TTCGTCGAGA TCTCGATCAG GAAGGTGGTC AGCACCCTGC TCGAGGCGAT GCTGCTGGTG TTCGCCGTGA TGTACCTGTT CATGCAGAAC TTCCGCGCCA CCCTGATCCC GACACTGGTG GTGCCGGTGG CCCTGCTGGG CACCTTCACG GTGATGCTCG GCCTGGGCTT CTCGATCAAC GTGCTGACCA TGTTCGGCAT GGTCCTGGCG ATCGGCATCC TGGTGGACGA CGCGATCATC GTGGTGGAGA ACGTCGAGCG GCTGATGGCC GAGGAAGGCC TGTCGCCGCA CGACGCCACG GTCAAGGCGA TGCGCCAGAT CAGCGGGGCC ATCGTCGGCA TCACCGTAGT GCTGGTCTCG GTGTTCGTGC CGATGGCGTT CTTCAGCGGC GCGGTGGGCA ACATCTACCG CCAGTTCGCG GTGACCCTGG CGGTCTCCAT CGGCTTCTCG GCGTTCCTCG CGCTGTCGCT GACCCCGGCC CTGTGCGCCA CCCTGCTGCG CCCGATCGAC GCCGACCACC ACGAGAAGCG CGGCTTCTTC GGCTGGTTCA ACCGCGCCTT CCTGCGCCTG ACCGGACGCT ACCGCAACGC GGTGGCCGGC ATCCTCGCCC GGCCGATCCG CTGGATGCTG GTCTACACCC TGGTCATCGG CGTGGTCGCC CTGCTCTTCG TGCGCCTGCC GCAGGCGTTC CTGCCGGAAG AGGACCAGGG CGACTTCATG ATCATGGTGA TGCAGCCCGA AGGCACGCCG ATGGCGGAGA CCATGGCCAA CGTCGGCGAC GTCGAGCGCT ACCTGGCGGA GCACGAACCG GTGGCCTACG CCTATGCGGT CGGCGGCTTC AGCCTGTACG GCGACGGCAC CAGCTCGGCG ATGATCTTCG CCACCCTGAA GGACTGGTCG GAACGCCGGG AGGCCAGCCA GCACGTCGGC GCCATCGTCG AGCGCATCAA CCAGCGCTTC GCCGGCCTGC CCAACCGTAC 


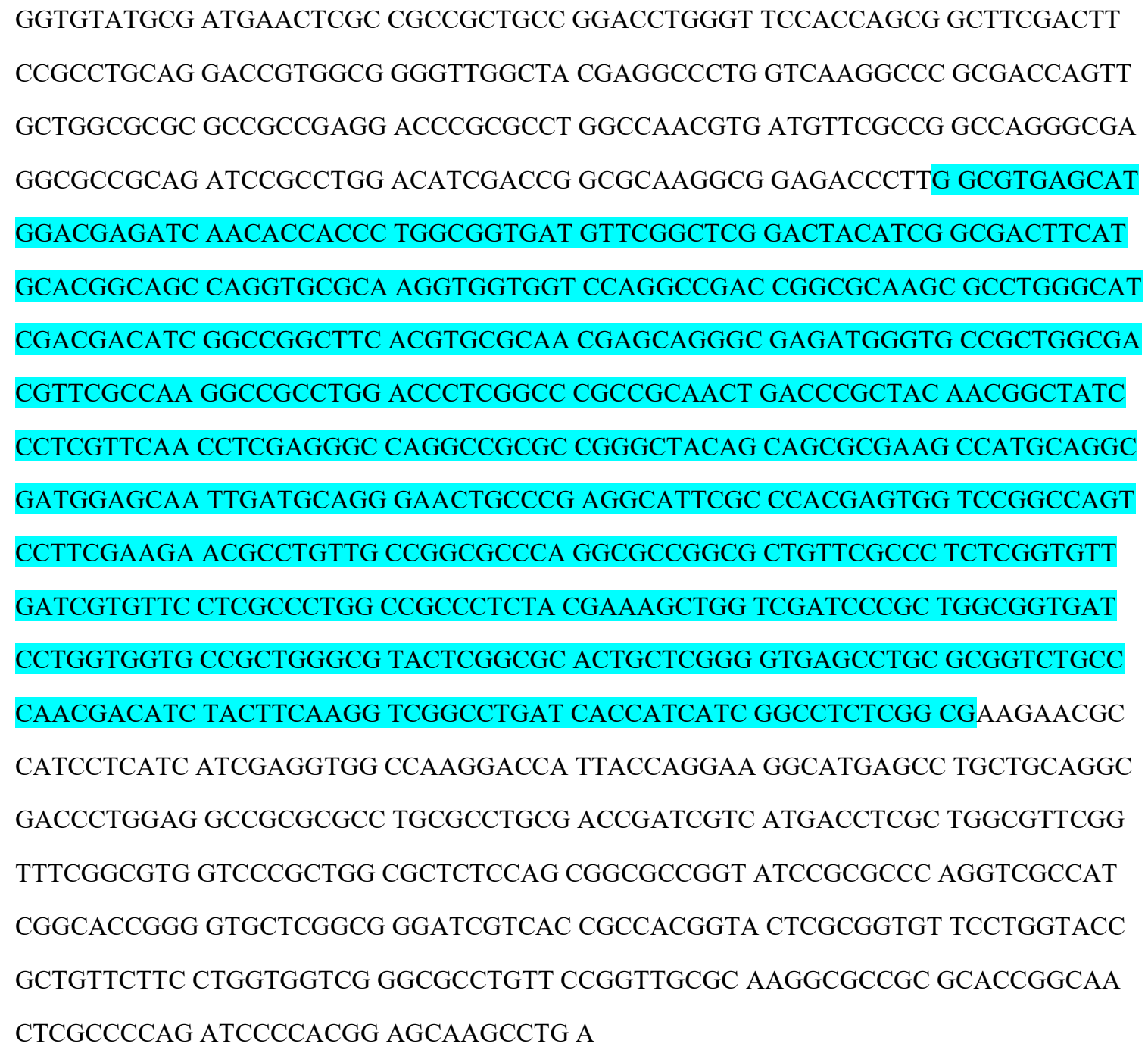

Figure S2. MexY gene coding sequence. The two amplicons used in sequence comparison are highlighted in green (270 bp) and blue (588 bp), respectively.

MARFFIDRPVFAWVISLLIVLAGVLAIRFLPVAQYPDIAPPVVNVSATYPGASAKVVEEAVTAIIER EMNGAPGLLYTKATSSTGQASLTLTFRQGVNANLAAVEVQNRLKIVESRLPESVRRDGIYVEKA ADSIQLIVTLTSSSGRYDAMELGEIASSNVLQALRRVEGVGKVETWGAEYAMRIWPDPAKLTSM NLSASDLVNAVRRHNARLTVGDIGNLGVPDSAPISATVKVDDTLVTPEQFGEIPLAHPRDGGAIR LRDVARVEFGQSEYGFVSRVNQMTATGLAVKMAPGSNAVATAKRIRATLDELSRYFPEGVSYNI PYDTSAFVEISIRKVVSTLLEAMLLVFAVMYLFMQNFRATLIPTLVVPVALLGTFTVMLGLGFSIN VLTMFGMVLAIGILVDDAIIVVENVERLMAEEGLSPHDATVKAMRQISGAIVGITVVLVSVFVPM AFFSGAVGNIYRQFAVTLAVSIGFSAFLALSLTPALCATLLRPIDADHHEKRGFFGWFNRAFLRLT GRYRNAVAGILARPIRWMLVYTLVIGVVALLFVRLPQAFLPEEDQGDFMIMVMQPEGTPMAET MANVGDVERYLAEHEPVAYAYAVGGFSLYGDGTSSAMIFATLKDWSERREASQHVGAIVERIN QRFAGLPNRTVYAMNSPPLPDLGSTSGFDFRLQDRGGVGYEALVKARDQLLARAAEDPRLANV MFAGQGEAPQIRLDIDRRKAETLGVSMDEINTTLAVMFGSDYIGDFMHGSQVRKVVVQADRRK RLGIDDIGRLHVRNEQGEMGAAGDVRQGRLDPRPAATDPLQRLSLVQPRGPGRAGLQQREAMQ AMEQLMQGTARGIRPRVVRPVLRRTPVAGAQAPALFALSVLIVFLALAALYESWSIPLAVILVVP LGVLGALLGVSLRGLPNDIYFKVGLITIIGLSAKNAILIIEVAKDHYQEGMSLLQATLEAARLRLRP IVMTSLAFGFGVVPLALSSGAGIRAQVAIGTGVLGGIVTATVLAVFLVPLFFLVVGRLFRLRKAPR TGNSPQIPTEQA-

Figure S3. MexY amino acid sequences obtained from the conversion of nucleotide sequences. The regions corresponding to the two selected amplicons are highlighted in green (270 bp) and blue (588 bp), respectively. 


\section{Sequence 1 (270 bp)}

PAO1

C5 9

C8 4

C25

C31

AR 48

C5 4

C30

NCO 6

$\mathrm{PAO} 1$

C5 9

C8 4

C25

C31

AR 48

C5 4

C30

NCO 6

PAO1

C59

C8 4

C25

C31

AR 48

C5 4

C30

NCO 6

PAO1

C5 9

C8 4

C25

C31

AR 48

C5 4

C30

NC0 6

TGGAAGTGCAGAACCGCCTGAAGATCGTCGAGTCGCGCCTGCCCGAATCGGTGCGGCGCG 60 -------------------CTACGTCGGTCGCGCCTGCCCGAATCGGTGCGGCGCG 37 --------------------TGTACGTCGGTCGCGCCTGCCCGAATCGGTGCGGCGCG 38 --------------------TGTACGTCGGTCGCGCCTGCCCGAATCGGTGCGGCGCG 38 ---------------------CGTACTCGGTCGCGCCTGCCCGATCGGTGCGGCGCG 36 ---------------------CGTACGTCGATCGCGCCTGCTCCGATCGGTGCGGCGCG 38 ---------------------GGTACGTCGATCGC-GCCTGCCCGATCGGTGCGGCGCG 37 --------------------CGTACGTCGATCGC-GCCTGCCCGATCGGTGCGGCGCG 37 -CGTACGTCGATCGC-GCCTGCCCGATCGGTGCGGCGCG 37

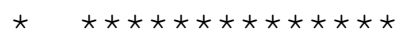

ACGGCATCTACGTGGAGAAGGCGGCGGACAGCATCCAGCTGATCGTTACCCTTACCTCCT 120 ACGGCATCTACGTGGAGAAGGCGGCGGACAGCATCCAGCTGATCGTTACCCTTACCTCCT 97 ACGGCATCTACGTGGAGAAAGCGGCGGACAGCATCCAGCTGATCGTTACCCTTACCTCCT 98 ACGGCATCTACGTGGAGAAGGCGGCGGACAGCATCCAACTGATCGTTACCCTTACCTCCT 98 ACGGCATCTATGTGGAGAAGGCGGCGGACAGCATCCAACTGATCGTCACCCTTACCTCCT 96 ACGGCATCTATGTGGAGAAGGCGGCGGACAGCATCCAACTGATCGTCACCCTTACCTCCT 98 ACGGCATCTACGTGGAGAAGGCGGCGGACAGCATCCAGCTGATCGTTACCCTTACCTCCT 97 ACGGCATCTATGTGGAGAAGGCGGCGGACAGCATCCAACTGATCGTCACCCTTACCTCCT 97 ACGGCATCTATGTGGAGAAGGCGGCGGACAGCATCCAGCTGATCGTCACCCTCACCTCCT 97

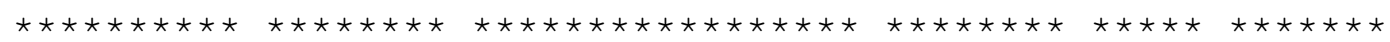

CCAGCGGCCGCTACGACGCCATGGAGCTGGGCGAGATCGCCTCGTCCAACGTGTTGCAGG 180 CCAGCGGCCGCTACGACGCCATGGAGCTGGGCGAGATCGCCTCGTCCAACGTGTTGCAGG 157 CCAGCGGCCGCTACGACGCCATGGAGCTGGGCGAGATCGCCTCGTCCAACGTGTTGCAGG 158 CCAGCGGCCGCTACGACGCCATGGAGCTGGGCGAGATCGCCTCGTCCAACGTGTTGCAGG 158 CCAGCGGCCGCTACGACGCCATGGAGCTGGGCGAGATCGCCTCGTCCAACGTGTTGCAGG 156 CCAGCGGCCGCTACGACGCCATGGAGCTGGGCGAGATCGCCTCGTCCAACGTGTTGCAGG 158 CCAGCGGCCGCTACGACGCCATGGAGCTGGGCGAGATCGCCTCGTCCAACGTGTTGCAGG 157 CCAGCGGCCGCTACGACGCCATGGAGCTGGGCGAGATCGCCTCGTCCAACGTGTTGCAGG 157 CCAGCGGCCGCTACGACGCCATGGAACTGGGCGAGATCGCCTCGTCCAACGTGTTGCAGG 157

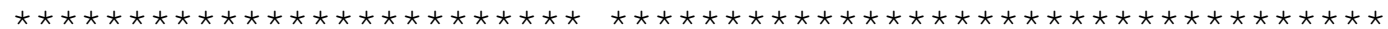

CGCTGCGCCGGGTGGAGGGCGTGGGCAAGGTCGAGACCTGGGGCGCCGAGTACGCCATGC 240 CGCTGCGCCGGGTGGAGGGCGTGGGCAAGGTCGAGACCTGGGGCGCCGAGTACGCCATGC 217 CGCTGCGCCGGGTGGAGGGCGTGGGCAAGGTCGAGACCTGGGGCGCCGAGTACGCCATGC 218 CGCTGCGCCGGGTGGAGGGCGTGGGCAAGGTCGAGACCTGGGGCGCCGAGTACGCCATGC 218 CGCTGCGCCGGGTGGAGGGCGTGGGCAAGGTCGAGACCTGGGGCGCCGAGTACGCCATGC 216 CGCTGCGCCGGGTGGAGGGCGTGGGCAAGGTCGAGACCTGGGGCGCCGAGTACGCCATGC 218 CGCTGCGCCGGGTGGAGGGCGTGGGCAAGGTCGAGACCTGGGGCGCCGAGTACGCCATGC 217 CGCTGCGCCGGGTGGAGGGCGTGGGCAAGGTCGAGACCTGGGGCGCCGAGTACGCCATGC 217 CGCTGCGCCGGGTGGAGGGCGTGGGCAAGGTCGAGACCTGGGGCGCCGAGTACGCCATGC 217

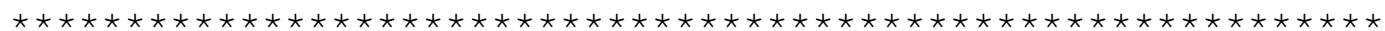




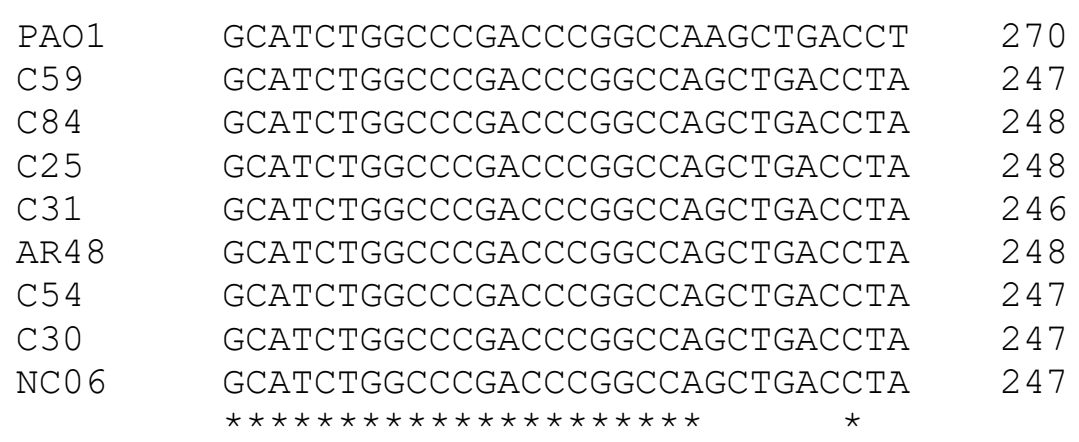

\section{Sequence 2 (588 bp)}

PAO1

C30

C59

C25

C5 4

C84

NCO 6

AR 48

C31

$\mathrm{PAO} 1$

C30

C5 9

C25

C5 4

$\mathrm{C} 84$

NC0 6

AR4 8

C31

PAO1

C30

C5 9

C25

C5 4

C8 4

NC0 6

AR 48

C31

CGTGAGCATGGACGAGATCAACACCACCCTGGCGGTGATGTTCGGCTCGGACTACATCGG 60 -----------------------CCTGGCGGTGATGTTCGGCTCGGACTACATCGG 33 ----------------------CCCTGGCGGTGATGTTCGGCTCGGACTACATCGG 34 -------------------CCCCCCTGGCGGTGATGTTCGGCTCGGACTACATCGG 37 --------------------------CCCTGGCGGTGATGTTCGGCTCGGACTACATCGG 34 ------------------------CCTGGCGGTGATGTTCGGCTCGGACTACATCGG 33 ----------------------------GCGGTGATGTTCGGCTCGGACTACATCGG 29 -CCTGGCGGTGATGTTCGGCTCGGACTACATCGG 33 -CCCTGGCGGTGATGTTCGGCTCGGACTACATCGG 34

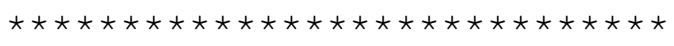

CGACTTCATGCACGGCAGCCAGGTGCGCAAGGTGGTGGTCCAGGCCGACCGGCGCAAGCG 120 CGACTTCATGCACGGCAGCCAGGTGCGCAAGGTGGTGGTCCAGGCCGACGGCGCCAAGCG 93 CGACTTCATGCACGGCAGCCAGGTGCGCAAGGTGGTGGTCCAGGCCGACGGCGCCAAGCG 94 CGACTTCATGCACGGCAGCCAGGTGCGCAAGGTGGTGGTCCAGGCCGACGGCGCCAAGCG 97 CGACTTCATGCACGGCAGCCAGGTGCGCAAGGTGGTGGTCCAGGCCGACGGCGCCAAGCG 94 CGACTTCATGCACGGCAGCCAGGTGCGCAAGGTGGTGGTCCAGGCCGACGGCACCAAGCG 93 CGACTTCATGCACGGCAGCCAGGTGCGCAAGGTGGTGGTCCAGGCCGACGGCGCCAAGCG 89 CGACTTCATGCACGGCAGCCAGGTGCGCAAGGTGGTGGTCCAGGCCGACGGCGCCAAGCG 93 CGACTTCATGCACGGCAGCCAGGTGCGCAAGGTGGTGGTCCAGGCCGACGGCGCCAAGCG 94

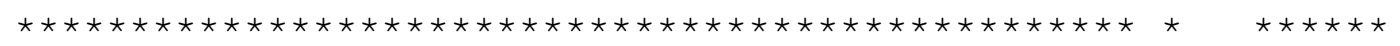

CCTGGGCATCGACGACATCGGCCGGCTTCACGTGCGCAACGAGCAGGGCGAGATGGGTGC 180 CCTGGGCATCGACGACATCGGCCGGCTTCACGTGCGCAACGAGCAGGGCGAGATG-GTCC 152 CCTGGGCATCGACGACATCGGCCGGCTTCACGTGCGCAACGAGCAGGGCGAGATG-GTCC 153 CCTGGGCATCGACGACATCGGCCGGCTTCACGTGCGCAACGAGCAGGGCGAGATG-GTGC 156 CCTGGGCATCGACGACATCGGCCGGCTTCACGTGCGCAACGAGCAGGGCGAGATG-GTGC 153 CCTGGGCATCGACGACATCGGCCGGCTTCACGTGCGCAACGAGCAGGGCGAGATG-GTGC 152 CCTGGGCATCGACGACATCGGCCGGCTTCACGTGCGCAACGAGCAGGGCGAGATG-GTCC 148 CCTGGGCATCGACGACATCGGCCGGCTTCACGTGCGCAACGAGCAGGGCGAGATG-GTCC 152 CCTGGGCATCGACGACATCGGCCGGCTTCACGTGCGCAACGAGCAGGGCGAGATG-GTCC 153

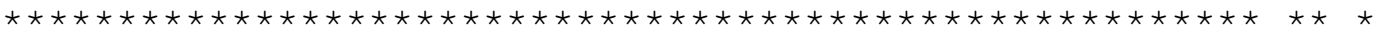


PAO1

C30

C5 9

C25

C5 4

C8 4

NC0 6

AR 48

C31

PAO1

C30

C5 9

C25

C5 4

C8 4

$\mathrm{NC} 06$

AR 48

C31

PAO1

C30

C59

C25

C5 4

C8 4

$\mathrm{NC} 06$

AR 48

C31

PAO1

C30

C59

C25

C5 4

C8 4

NC0 6

AR 48

C31

PAO1

C 30

C59

C25

C5 4

C8 4

$\mathrm{NC} 06$

AR 48

C31

CGCTGGCGACGTTCGCCAAGGCCGCCTGGACCCTCGGCCCGCCGCAACTGACCCGCTACA 240 CGCTGGCGACGTTCGCCAAGGCCGCCTGGACCCTCGGCCCGCCGCAACTGACCCGCTACA 212 CGCTGGCGACGTTCGCCAAGGCCGCCTGGACCCTCGGCCCGCCGCAACTGACCCGCTACA 213 CGCTGGCGACGTTCGCCAAGGCCGCCTGGACCCTCGGCCCGCCGCAACTGACCCGCTACA 216 CGCTGGCGACGTTCGCCAAGGCCGCCTGGACCCTCGGCCCGCCGCAACTGACCCGCTACA 213 CGCTGGCGACGTTCGCCAAGGCCGCCTGGACCCTCGGCCCGCCGCAACTGACCCGCTACA 212 CGCTGGCGACGTTCGCCAAGGCCGCCTGGACCCTCGGCCCGCCGCAACTGACCCGCTACA 208 CGCTGGCGACGTTCGCCAAGGCCGCCTGGACCCTCGGCCCGCCGCAACTGACCCGCTACA 212 CGCTGGCGACGTTCGCCAAGGCCGCCTGGACCCTCGGCCCGCCGCAACTGACCCGCTACA 213

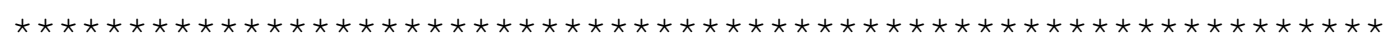

ACGGCTATCCCTCGTTCAACCTCGAGGGCCAGGCCGCGCCGGGCTACAGCAGCGCGAAG- 299 ACGGCTATCCCTCGTTCAACCTCGAGGGCCAGGCCGCGCCGGGCTACAGCAGCGGCGAAG 272 ACGGCTATCCCTCGTTCAACCTCGAGGGCCAGGCCGCGCCGGGCTACAGCAGCGGCGAAG 273 ACGGCTATCCCTCGTTCAACCTCGAGGGCCAGGCCGCGCCGGGCTACAGCAGCGGCGAAG 276 ACGGCTATCCCTCGTTCAACCTCGAGGGCCAGGCCGCGCCGGGCTACAGCAGCGGCGAAG 273 ACGGCTATCCCTCGTTCAACCTCGAGGGCCAGGCCGCGCCGGGCTACAGCAGCGGCGAAG 272 ACGGCTATCCCTCGTTCAACCTCGAGGGCCAGGCCGCGCCGGGCTACAGCAGCGGCGAAG 268 ACGGCTATCCCTCGTTCAACCTCGAGGGCCAGGCCGCGCCGGGCTACAGCAGCGGCGAAG 272 ACGGCTATCCCTCGTTCAACCTCGAGGGCCAGGCCGCGCCGGGCTACAGCAGCGGCGAAG 273

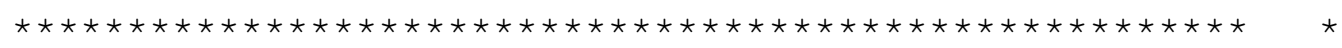

CCATGCAGGCGATGGAGCAATTGATGCAGGGAACTGCCCGAGGCATTCGCCCACGAGTGG 359 CCATGCAGGCGATGGAGGAATTGATGCA-GGGCCTGCCCGAGGGCATCGCCCACGAGTGG 331 CCATGCAGGCGATGGAGGAATTGATGCA-GGGCCTGCCCGAGGGCATCGCCCACGAGTGG 332 CCATGCAGGCGATGGAGGAATTGATGCA-GGGCCTGCCCGAGGGCATCGCCCACGAGTGG 335 CCATGCAGGCGATGGAGCAATTGATGCA-GGGACTGCCCGAGGGCATCGCCCACGAGTGG 332 CCATGCAGGCGATGGAGGAATTGATGCA-GGGCCTGCCCGAGGGCATCGCCCACGAGTGG 331 CCATGCAGGCGATGGAGCAATTGATGCA-GGGCCTGCCCGAGGGCATCGCCCACGAGTGG 327 CCATGCAGGCGATGGAGGAATTGATGCA-GGGCCTGCCCGAGGGCATCGCCCACGAGTGG 331 CCATGCAGGCGATGGAGGAATTGATGCA-GGGCCTGCCCGAGGGCATCGCCCACGAGTGG 332

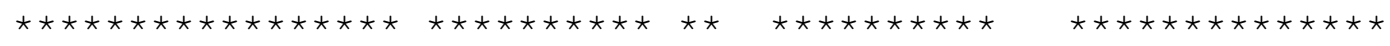

TCCGGCCAGTCCTTCGAAGAACGCCTGTTGCCGGCGCCCAGGCGCCGGCGCTGTTCGCCC 419 TCCGGCCAGTCCTTCGAAGAACGCCTGTC--CGGCGCCCAGGCGCCGGCGCTGTTCGCCC 389 TCCGGCCAGTCCTTCGAAGAACGCCTGTC--CGGCGCCCAGGCGCCGGCGCTGTTCGCCC 390 TCCGGCCAGTCCTTCGAAGAACGCCTGTC--CGGCGCCCAGGCGCCGGCGCTGTTCGCCC 393 TCCGGCCAGTCCTTCGAAGAACGCCTGTC--CGGCGCCCAGGCGCCGGCGCTGTTCGCCC 390 TCCGGCCAGTCCTTCGAAGAACGCCTGTC--CGGCGCCCAGGCGCCGGCGCTGTTCGCCC 389 TCCGGCCAGTCCTTCGAAGAACGCCTGTC--CGGCGCCCAGGCGCCGGCGCTGTTCGCCC 385 TCCGGCCAGTCCTTCGAAGAACGCCTGTC--CGGCGCCCAGGCGCCGGCGCTGTTCGCCC 389 TCCGGCCAGTCCTTCGAAGAACGCCTGTC--CGGCGCCCAGGCGCCGGCGCTGTTCGCCC 390

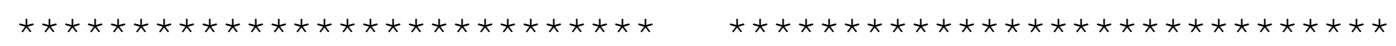

TCTCGGTGTTGATCGTGTTCCTCGCCCTGGCCGCCCTCTACGAAAGCTGGTCGATCCCGC 479 TCTCGGTGCTGATCGTGTTCCTCGCCCTGGCCGCCCTCTACGAAAGCTGGTCGATCCCGC 449 TCTCGGTGTTGATCGTGTTCCTCGCCCTGGCGGCCCTCTACGAAAGCTGGTCGATCCCGC 450 TCTCGGTGTTGATCGTGTTCCTCGCCCTGGCCGCCCTCTACGAAAGCTGGTCGATCCCGC 453 TCTCGGTGTTGATCGTGTTCCTCGCCCTGGCCGCCCTCTACGAAAGCTGGTCGATCCCGC 450 TCTCGGTGTTGATCGTGTTCCTCGCCCTGGCCGCCCTCTACGAAAGCTGGTCGATCCCGC 449 TCTCGGTGCTGATCGTGTTCCTCGCCCTGGCCGCCCTCTACGAAAGCTGGTCGATCCCGC 445 TCTCGGTGCTGATCGTGTTCCTCGCCCTGGCCGCCCTCTACGAAAGCTGGTCGATCCCGC 449 TCTCGGTGCTGATCGTGTTCCTCGCCCTGGCCGCCCTCTACGAAAGCTGGTCGATCCCGC 450

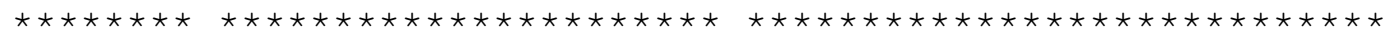


TGGCGGTGATCCTGGTGGTGCCGCTGGGCGTACTCGGCGCACTGCTCGGGGTGAGCCTGC 539

C30 TGGCGGTGATCCTGGTGGTGCCGCTGGGCGTGCTCGGCGCAGCTGCACGGGAGTGA---- 505 TGGCGGTGATCCTGGTGGTGCCGCTGGGCGTACTCGGCGCACTGCTCGGGGTGAGCCTGC 510 TGGCGGTGATCCTGGTGGTGCCGCTGGGCGTACTCGGCGCACTGCTCGGGGTGAGCCTGC 513 TGGCGGTGATCCTGGTGGTGCCGCTGGGCGTACTCGGCGCACTGCTCGGGGTGAGCCTGC 510 TGGCGGTGATCCTGGTGGTGCCGCTGGGCGTACTCGGCGCACTGCTCGGGGTGAGCCTGC 509 TGGCGGTGATCCTGGTGGTGCCGCTGGGCGTGCTCGGCGCACTGCTCGGGGTGAGCCTGC 505 TGGCGGTGATCCTGGTGGTGCCGCTGGGCGTGCTCGGCGCACTGCTCGGGGTGAGCCTGC 509

C31 TGGCGGTGATCCTGGTGGTGCCGCTGGGCGTGCTCGGCGCACTGCTCGGGGTGAGCCTGC 510

PAO1

C30

C59

C25

C5 4

C8 4

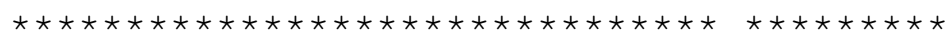
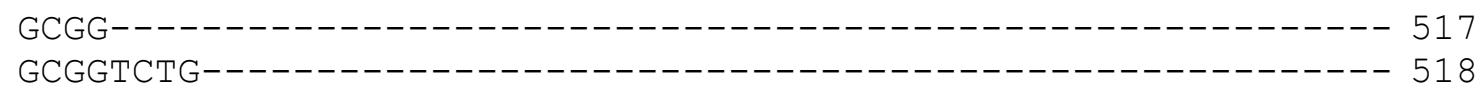

C31

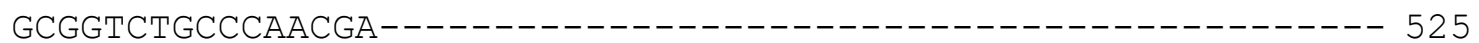

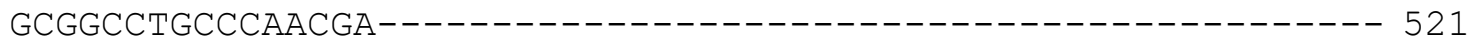

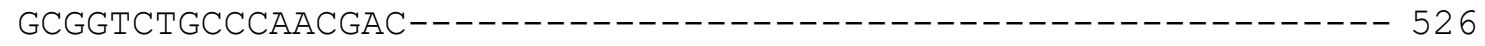

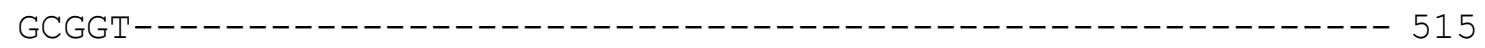

Figure S4. MexY multiple sequence alignment. Alignment of the nucleotide sequences of two amplicons of the eight $P$. aeruginosa strains selected for polymorphism analysis and corresponding sequences of $P$. aeruginosa PAO1.

\begin{tabular}{|c|c|}
\hline & $\ldots \ldots{ }^{10} \ldots \ldots \ldots{ }^{30} \ldots \ldots \ldots \ldots$ \\
\hline PA01 & MARFFIDRPVFAVVISLLIVLAGVLAIRFLPVAQYPDIAPPVVNVSATYPGASARVVEEAVTAIIEREMNGAPGL \\
\hline AR48 & 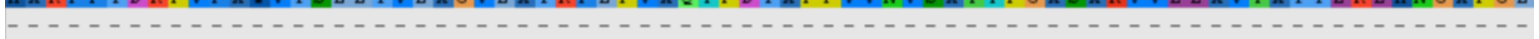 \\
\hline C31 & 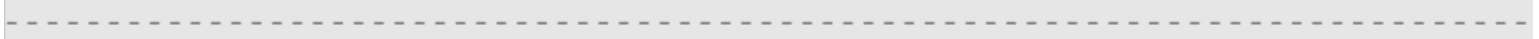 \\
\hline C59 & 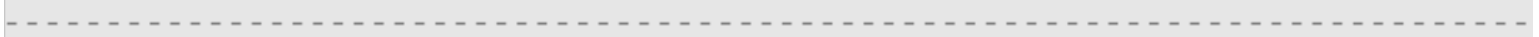 \\
\hline C54 & 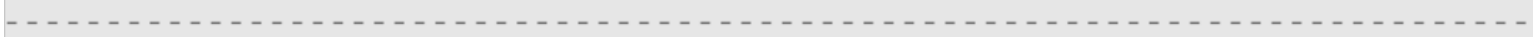 \\
\hline C84 & - - - - - \\
\hline C30 & 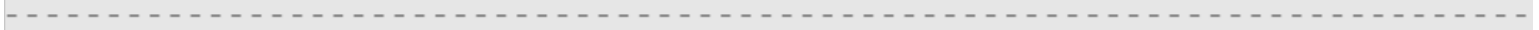 \\
\hline C25 & 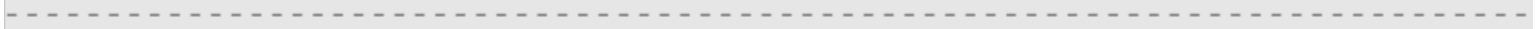 \\
\hline \multirow[t]{2}{*}{ NC06 } & 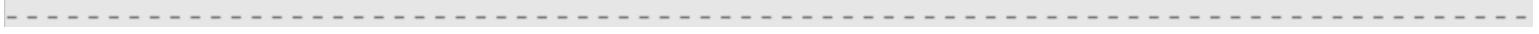 \\
\hline & 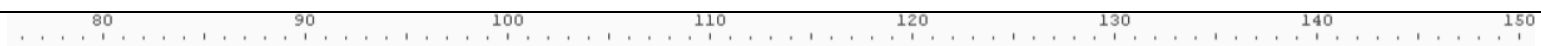 \\
\hline PA01 & LYTKATSSTGQASLTLTFRQGVNANLAAVEVQNRLKIVESRLPESVRRDGIYVEKAADIQLIVTLTSSSGR DA \\
\hline AR48 & . \\
\hline C31 & 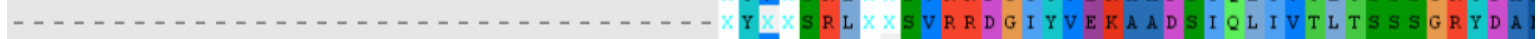 \\
\hline C59 & . \\
\hline C54 & 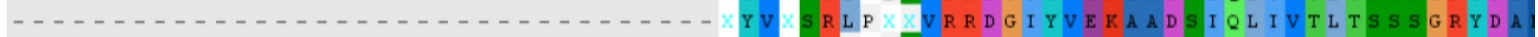 \\
\hline C84 & 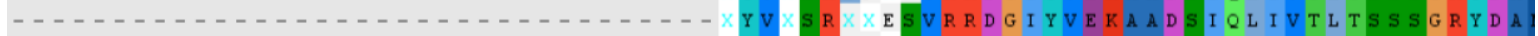 \\
\hline C30 & 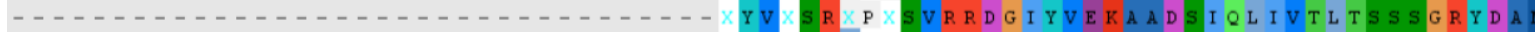 \\
\hline C25 & $\ldots \ldots \ldots \ldots \ldots \ldots$ Y X S R L X X S V R R D G I Y V E K A A D I Q L I V T L T S S S G R Y A \\
\hline \multirow[t]{2}{*}{ NC06 } & 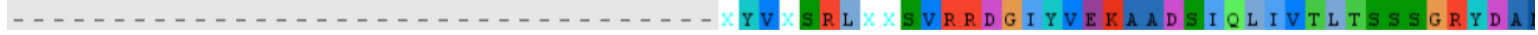 \\
\hline & 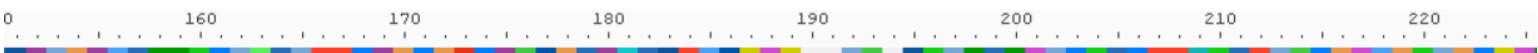 \\
\hline PA01 & 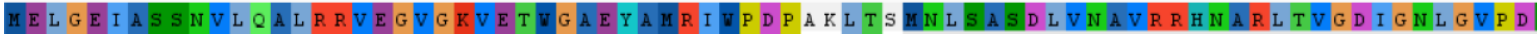 \\
\hline AR48 & MELGE I A S SNVL Q A L R RVEGVGKVET VG A E Y A M R I V P D P X X L T X $\ldots \ldots \ldots$ \\
\hline C31 & 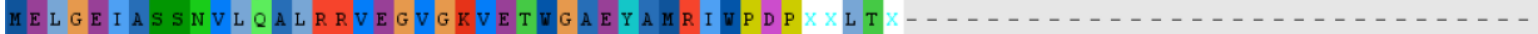 \\
\hline C59 & 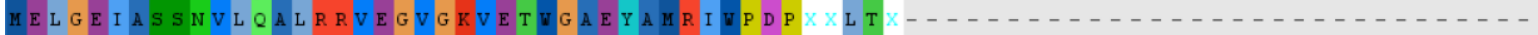 \\
\hline C54 & MEL GE I A S SNVL Q A L R RVEGVGKVETVG A E Y A M R I V P D P XX L T X \\
\hline C84 & M E L G E I A S NVL Q A L R R E GVGKVETVG A E Y A M R I V P D P X X L T X \\
\hline с 30 & MEL G E I S SNVL Q A L R RVEGVGKVETVG A E Y A M R I V P D P X X L T X \\
\hline C25 & MEL G E I S SNVL Q A L R R VEGVGKVET V G A E Y A M R I V P D P X X L T X $\ldots \ldots$ \\
\hline \multirow[t]{2}{*}{ NC06 } & MEL G E I A S SNVL Q A L R R VEGVGKV E T W G A E Y A M R I U P D P X X L T X $\ldots \ldots$ \\
\hline & 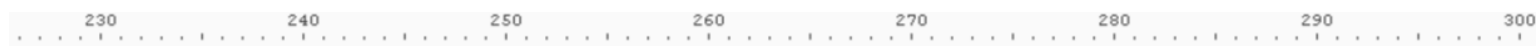 \\
\hline PA01 & SAPISATVKVDDTLVTPEQFGEIPLAHPRDGGAIRLRDVARVEFGQSEYGFVSRVNQMTATGLAVKMAPGSNAVA \\
\hline AR48 & 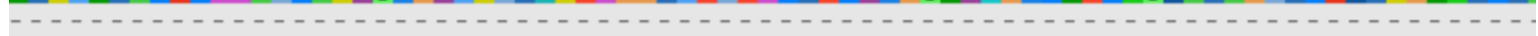 \\
\hline C31 & 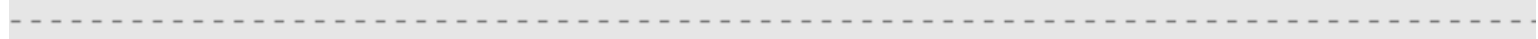 \\
\hline C59 & - - - - - - - - - - - - - - - - - - - - - - - - - - - - - - - - - - - - - - - - - - - - - \\
\hline C54 & 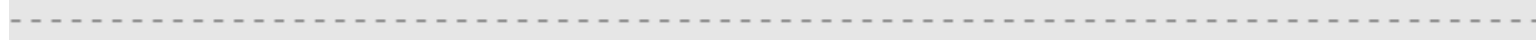 \\
\hline C84 & $\begin{array}{l}- \\
\end{array}$ \\
\hline C30 & 品 \\
\hline C25 & $\begin{array}{l}\text { } \\
\end{array}$ \\
\hline NC06 & $\begin{array}{l}{ }^{\prime} \\
\end{array}$ \\
\hline
\end{tabular}




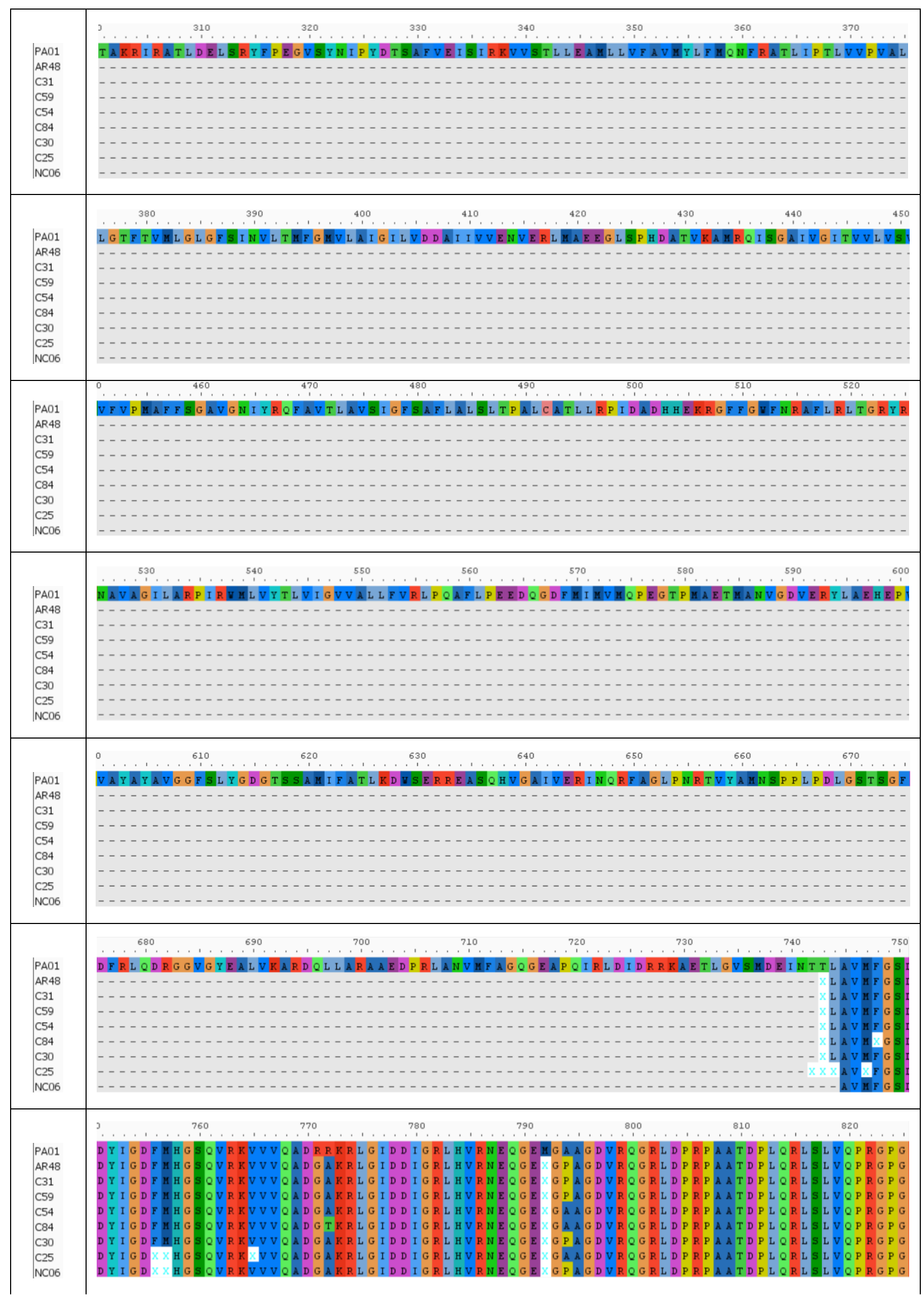




\begin{tabular}{|c|c|}
\hline & ${ }^{830} \ldots \ldots$ \\
\hline PA01 & 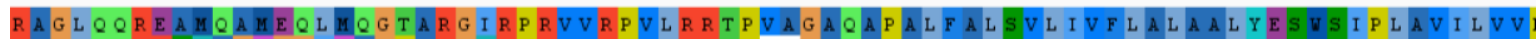 \\
\hline AR48 & 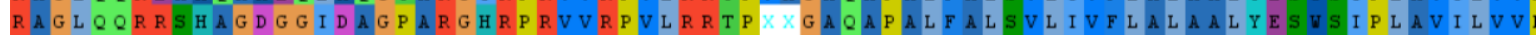 \\
\hline C31 & 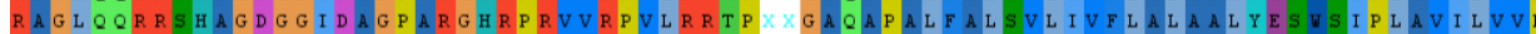 \\
\hline C59 & 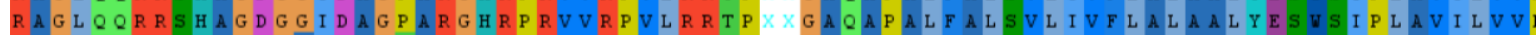 \\
\hline C54 & 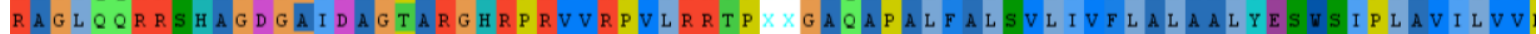 \\
\hline C84 & 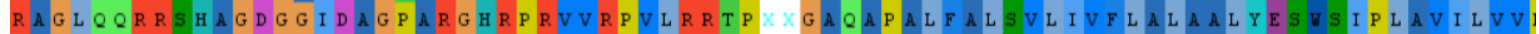 \\
\hline сзо & 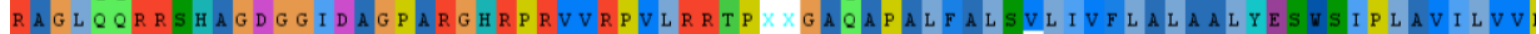 \\
\hline $\mathrm{C} 25$ & 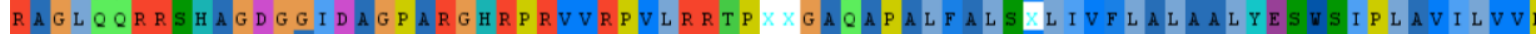 \\
\hline \multirow[t]{2}{*}{ NC06 } & RAGLQQRRSHAGDGAIDAGPARGHRPRVVRPVLRRTPXXGAQAPALFALSVLIVFLALAALYESWSIPLAVILVVA \\
\hline & 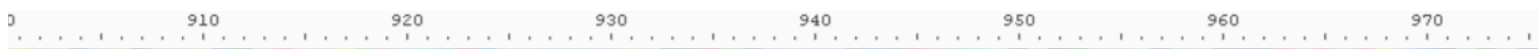 \\
\hline PA01 & 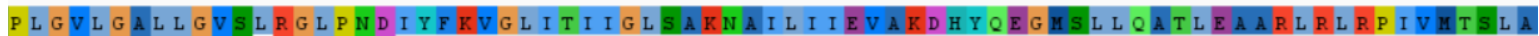 \\
\hline AR48 & 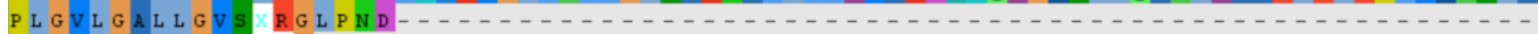 \\
\hline C31 & P L G V L G A L L G V S XXG \\
\hline C59 & 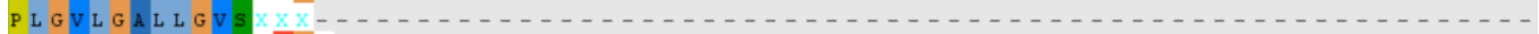 \\
\hline C54 & P L G VL G A L L G V S X R GX \\
\hline C84 & P L G VL G A L L G V SL R GXXNX \\
\hline C30 & 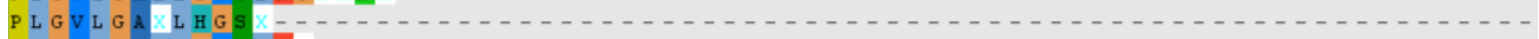 \\
\hline $\mathrm{C} 25$ & 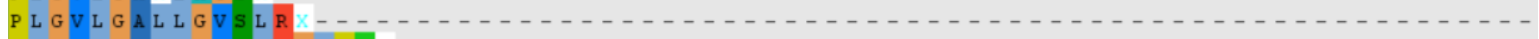 \\
\hline \multirow[t]{3}{*}{ NC06 } & P L G V L G A L L G V S L R G L P N X $\ldots \ldots \ldots \ldots$ \\
\hline & 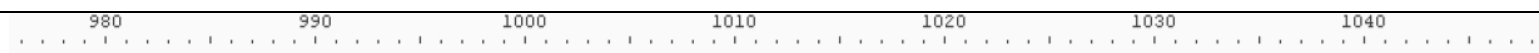 \\
\hline & FGFGVVPLALSSGAGIRAQVAIGTGVLGGIVTATVLAVFLVPLFFLVVGRLFRLRAPRTNSPQIPTEQA- \\
\hline PA01 & 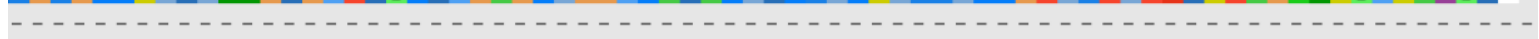 \\
\hline AR48 & 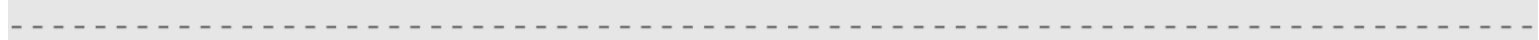 \\
\hline C31 & 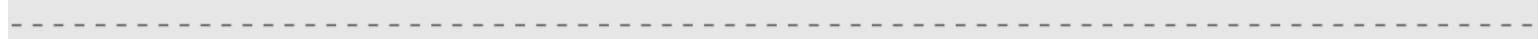 \\
\hline C59 & 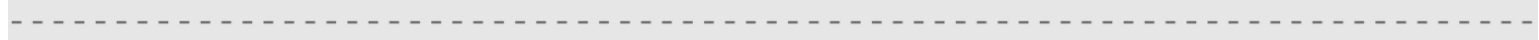 \\
\hline $\begin{array}{l}\text { C54 } \\
\text { C84 }\end{array}$ & 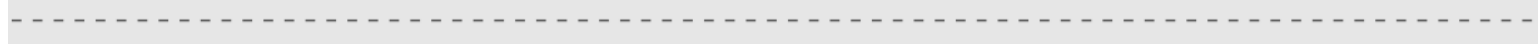 \\
\hline $\mathrm{C} 30$ & $-\cdots-$ \\
\hline C25 & 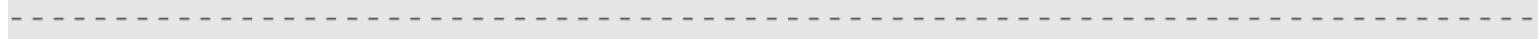 \\
\hline NC06 & - - - - - - - - - - - - - - - - - - - - - - - - - - - \\
\hline
\end{tabular}

Figure S5. MexY multiple sequence alignment. Alignment of the putative amino acid sequences retrieved from the corresponding nucleotide sequences of two amplicons of the eight $P$. aeruginos $a$ strains selected for polymorphism analysis and full MexY sequence of P. aeruginosa PAO1.
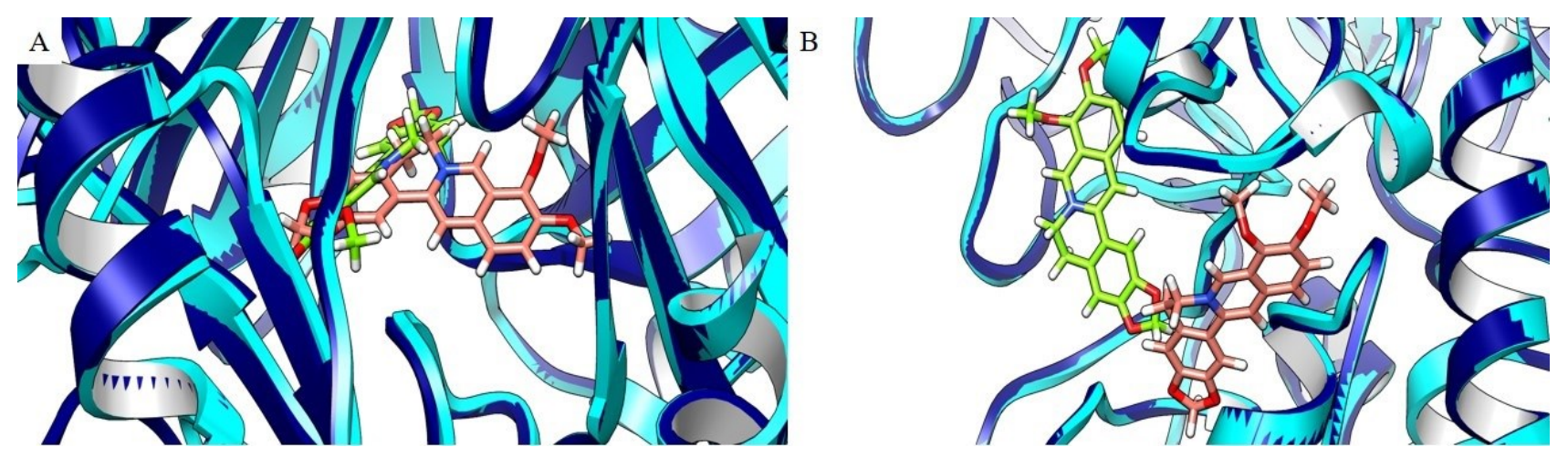

Figure S6. Binding mode of berberine to MexY to the antibiotic (A) and the cleft site (B). The overlapping MexY structures of $P$. aeruginosa $\mathrm{C} 31$ and $\mathrm{C} 59$ are shown in cyan and dark blue, respectively. Berberine molecules are shown in light green and brown in the binding model of $P$. aeruginosa $\mathrm{C} 31$ and $\mathrm{C} 59$, respectively. 
Table S1. Tobramycin resistance and persister reduction induced by berberine in 23 laboratory (L), CF and clinical (C) strains

\begin{tabular}{|c|c|c|}
\hline Bacterial strain & $\begin{array}{c}\text { MIC } \\
\text { reduction }\end{array}$ & $\begin{array}{l}\text { Persister } \\
\text { reduction }\end{array}$ \\
\hline P. aeruginosa $\mathrm{PAO} 1-\mathrm{T}(\mathrm{L})$ & 2 folds & $3 \log$ \\
\hline P. aeruginosa K767 (L) & 2 folds & $1 \log$ \\
\hline P. aeruginosa $\mathrm{PA} 14$ (L) & No & $1 \log$ \\
\hline P. aeruginosa C6 (CF) & 4 folds & $0.5 \log$ \\
\hline P. aeruginosa $\mathrm{C} 9(\mathrm{CF})$ & 2 folds & No \\
\hline P. aeruginosa $\mathrm{C} 15(\mathrm{CF})$ & No & $1 \log$ \\
\hline P. aeruginosa $\mathrm{C} 25(\mathrm{CF})$ & 16 folds & $2.5 \log$ \\
\hline P. aeruginosa $\mathrm{C} 30(\mathrm{CF})$ & No & $3 \log$ \\
\hline P. aeruginosa $\mathrm{C} 31(\mathrm{CF})$ & No & No \\
\hline P. aeruginosa $\mathrm{C} 54(\mathrm{C})$ & 2 folds & $1.5 \log$ \\
\hline P. aeruginosa C59(C) & 2 folds & No \\
\hline P. aeruginosa C61(C) & 4 folds & $7 \log$ \\
\hline P. aeruginosa $\mathrm{C} 76(\mathrm{C})$ & 2 folds & $2 \log$ \\
\hline P. aeruginosa $\mathrm{C} 84(\mathrm{C})$ & 4 folds & $0.5 \log$ \\
\hline P. aeruginosa $\mathrm{C} 95(\mathrm{C})$ & 8 folds & $1 \log$ \\
\hline P. aeruginosa $\mathrm{C} 104(\mathrm{C})$ & 4 folds & $2 \log$ \\
\hline P. aeruginosa AR48(CF) & 4 folds & No \\
\hline P. aeruginosa AR51(CF) & 2 folds & $1.5 \log$ \\
\hline P. aeruginosa $\mathrm{NC} 01(\mathrm{CF})$ & 2 folds & $2 \log$ \\
\hline P. aeruginosa $\mathrm{NC} 02(\mathrm{CF})$ & 2 folds & $1 \log$ \\
\hline P. aeruginosa $\mathrm{NC} 04(\mathrm{CF})$ & $>4$ folds & $1.5 \log$ \\
\hline P. aeruginosa $\mathrm{NC} 06(\mathrm{CF})$ & 4 folds & $2 \log$ \\
\hline P. aeruginosa $\mathrm{NC} 10(\mathrm{CF})$ & 4 folds & $1.5 \mathrm{log}$ \\
\hline
\end{tabular}

No, no reduction 